\title{
1 A Chemoenzymatic Method for Glycoproteomic N-glycan 2 Type Quantitation
}

3 Henghui $\mathrm{Li}^{1}$, Leyuan $\mathrm{Li}^{1}$, Kai Cheng ${ }^{1}$, Zhibin Ning ${ }^{1}$, Janice Mayne ${ }^{1}, \mathrm{Xu}$ Zhang ${ }^{1}$, Krystal Walker ${ }^{1}$,

4 Rui Chen ${ }^{3}$, Susan Twine ${ }^{3}$, Jianjun $\mathrm{Li}^{3}$, Daniel Figeys ${ }^{1,2^{*}}$

5

6

7

8

9

10

11

12

13

${ }^{1}$ SIMM-University of Ottawa Joint Research Center in Systems and Personalized Pharmacology and Ottawa Institute of Systems Biology and Department of Biochemistry, Microbiology and Immunology, Faculty of Medicine, University of Ottawa, 451 Smyth Road, Ottawa, ON, K1H 8M5, Canada.

${ }^{2}$ Molecular Architecture of Life Program, Canadian Institute for Advanced Research, Toronto, Canada.

${ }^{3}$ Human Health Therapeutics Research Centre, National Research Council Canada, Ottawa, ON, Canada.

*Corresponding authors

4 Ottawa Institute of Systems Biology and Department of Biochemistry, Microbiology and Immunology, Faculty of Medicine, University of Ottawa, 451 Smyth Road, Ottawa, ON, K1H 8M5, Canada.dfigeys@uottawa.ca. 


\section{ABSTRACT}

Glycosylation is one of the most important post-translational modifications in biological systems. Current glycoproteome methods mainly focus on qualitative identification of glycosylation sites or intact glycopeptides. However, the systematic quantitation of glycoproteins has remained largely unexplored. Here, we developed a chemoenzymatic method to quantitatively investigate N-glycoproteome based on the N-glycan types. Taking advantage of the specificity of different endoglycosidases and isotope dimethyl labeling, six N-glycan types of structures linked on each glycopeptide, including highmannose/hybrid, bi-antennary and tri-antennary with/without core fucose, were quantified. As a proof of principle, the glycoproteomic N-glycan type quantitative (glyco-TQ) method was first used to determine the N-glycan type composition of immunoglobulin G1 (IgG1) Fc fragment. Then we applied the method to analyze the glycan type profile of proteins in the breast cancer cell line MCF7, and quantitatively revealed the N-glycan type micro-heterogeneity at both the glycopeptide and glycoprotein levels. The novel quantitative strategy to evaluate the relative intensity of the six states of N-glycan type glycosylation on each site provides a new avenue to investigate function of glycoproteins in broad areas, such as cancer biomarker research, pharmaceuticals characterization and anti-glycan vaccine development.

Keywords: endoglycosidase; N-glycan; glycoproteomic; quantitation; isotope dimethyl labeling 


\section{INTRODUCTION}

Glycosylation is one of the most common post-translational modifications (PTM) ${ }^{1}$. Glycans exhibit vast structural microheterogeneity which is mainly generated by variable glycan structures at each of their specific glycosylation sites. The N-linked glycans are generally attached to the Asn at Asn-XSer/Thr consensus sequence, where $\mathrm{X}$ is any amino acid other than $\mathrm{Pro}^{2}$. The biosynthesis of N-linked glycoproteins is under a complex sequence of enzymatically catalyzed events, leading to a variety of diverse $\mathrm{N}$-glycan structures. The diverse $\mathrm{N}$-glycan structures are generally classified into three types: high mannose, hybrid, and complex type glycans, with all N-glycans sharing a common penta-saccharide $\left(\mathrm{GlcNAc}_{2} \mathrm{Man}_{3}\right)$ core structure ${ }^{3}$. Although the structure of glycan is variable, evidence shows that the mammalian glycans are remarkably well conserved in certain organisms, expressing a distinct array of glycan profiles under defined conditions ${ }^{4}$.

Mass spectrometry (MS) is a powerful platform to comprehensively analyze protein glycosylation. However, due to the low abundance of glycosylated peptides and the heterogeneity of glycan structures, $\mathrm{N}$-glycopeptide enrichment is required. Several enrichment methods have been reported, including lectin $^{5}$ and hydrazide chemistry-based methods ${ }^{6,7}$, boronic acid enrichment ${ }^{8}$, hydrophilic interaction liquid chromatography (HILIC) $)^{9}$ and metabolic labeling ${ }^{10,11}$. In general, these strategies for detecting Nlinked glycosylated sites require an additional de-glycosylation step by N-glycosidase F (PNGase F) before MS detection ${ }^{5-7}$. Unfortunately, this results in the loss of the glycan structure information at the glycosylated sites as the glycans are removed from the peptides.

Recently, a site-specific glycoproteomic method was used to detect intact glycopeptide by MS with a variety of tandem MS techniques ${ }^{10,12-14}$. This strategy allows the simultaneous detection of glycopeptide sequence, glycosylation site and glycan structures in one MS/MS spectrum. Current state-of-the-art MS technology with multiple dissociation known as activated ion electron transfer dissociation methods (AIETD) allowed intact glycoproteomic identification of more than one thousand $(\sim 1500)$ intact Nglycopeptides from a mouse brain tissue ${ }^{13}$. The site-specific glycoproteomic strategies have been applied to quantitatively detect glycoprotein alteration using the isotope labeling ${ }^{12}$, isobaric labeling strategies ${ }^{15}$ and labeling-free method ${ }^{16}$. However, due to missed detection of low abundant glycan structures, more than half of identified glycopeptides were linked with only one or two glycan structures using the intact glycopeptide method, hindering the comprehensive quantitation in complex biological samples ${ }^{13}$.

The diverse $\mathrm{N}$-glycan structures play important roles in many key biological processes, including cell 
adhesion, receptor activation, molecular trafficking, signal transduction and disease progression, and immunotherapy ${ }^{17,18}$. Some apparent changes associated with cancers are the overexpression of sialylation and core fucosylation, and complex branched N-glycans. For example, increased core fucosylated type of $\mathrm{N}$-glycan is an important signature of several cancers, such as hepatocellular carcinoma ${ }^{19}$, lung cancer $^{20}$ and breast cancer ${ }^{21}$. Therefore, quantitatively monitoring the $\mathrm{N}$-glycan type changes in glycosylation are important for the diagnosis, prognosis, and understanding molecular mechanisms involved in pathogenesis. Cao L et al. introduced a MS-based method that used two glycosidases PNGase F and endoglycosidase $\mathrm{H}$ (Endo-H), to assess the site occupancy and proportion of highmannose and complex-type glycans of purified human immunodeficiency virus (HIV) envelope (Env) glycoprotein ${ }^{22}$. The NMR-based strategy was introduced to allow dissecting the glycan pattern of the IgE high-affinity receptor (FcعRI $\alpha$ ), presenting of pauci-mannose, high-mannose, hybrid, and bi-, tri-, and tetra-antennary complex type $\mathrm{N}$-glycans with different degrees of fucosylation and sialylation ${ }^{23}$. A purification step of glycoprotein is required in these methods, therefore, glycan type quantitation at the proteome level is urgently needed for complex biological samples.

To fulfill this analytical challenge, we developed a robust chemoenzymatic based method that quantitatively determined the proportion of N-glycan types at each glycopeptide. Briefly, three aliquots of trypsin proteolyzed sample are treated in parallel with three specific endoglycosidases and the aliquots are isotopically labeled using the three plex dimethyl labeling strategy and then combined. The cleaved $\mathrm{N}$-glycopeptides are biotinylated and enriched by affinity chromatography, and the eluted $\mathrm{N}$ glycopeptides are analyzed by MS. The glycoproteomic N-glycan type quantitative (Glyco-TQ) strategy was first applied on the standard glycoprotein IgG1 Fc fragment and further used to comprehensively investigate glycopeptides from the MCF7 breast cancer cell line. The data interpretation is convenient and compatible with the general proteomic platform, without need of laboriously generating samplespecific spectral libraries, complex data filtering process or specialized commercial data analysis tools. The result showed that the novel strategy could provide quantitative information on important characteristic of glycoproteins, including the relative proportion of high-mannose and linkage-related complex type glycan, and proportion of non-fucosylated and core fucosylated type glycan at each glycosylated site. To our knowledge, this is the first report to quantify the proportion of $\mathrm{N}$-glycan types on the glycopeptides using the data-dependent acquisition mode for a complex biological sample. 


\section{RESULTS}

We developed a method for the quantitative analysis of N-glycan type micro-heterogeneity at both glycopeptide and glycoprotein levels. The method includes the following steps (Fig. 1a): (i) the trypsin proteolyzed peptides were divided into three aliquots, treated with one of three endoglycosidase $(\mathrm{H}, \mathrm{S}$ and F3), and incubated with $\beta$-N-acetylhexosaminidase $e_{\mathrm{f}}$ to remove O-linked $\mathrm{N}$-acetylglucosamines (OGlcNAc); Endoglycosidase H (Endo-H) releases high mannose and hybrid type N-glycans, including those that have a fucose residue attached to the core structure ${ }^{24}$; endoglycosidase $\mathrm{S}$ (Endo-S) releases biantennary complex type glycans ${ }^{25}$; and endoglycosidase F3 (Endo-F3) release core fucosylated biantennary complex type glycan and tri-antennary complex glycan from $\mathrm{N}$-glycopeptides ${ }^{26}$ (ii) the three aliquots were dimethyl labeled with 'light' isotope for Endo-H treated peptides, 'intermediate' isotope for Endo-S treated peptides and 'heavy' isotope for Endo-F3 treated peptides, respectively; (iii) the aliquots were combined and the retained GlcNAc on the N-glycopeptide was further transformed with $\mathrm{N}$-azidoacetylgalactosamine (GalcNAz) through the catalysis of $\beta$-1,4-galactosyltransferase Y289L (GalT1 Y298L); (iv) the GalNAz labeled N-glycopeptides were covalently reacted with the photocleavable (PC) alkyne biotin through the copper(I)-catalyzed azide-alkyne cycloaddition (CuAAC) reaction; (v) the biotin linked peptides were enriched by high capacity streptavidin agarose affinity chromatography. The non-glycopeptide and reagents were removed through extensively washing; (vi) the N-glycopeptides were released by $365 \mathrm{~nm}$ ultraviolet irradiation and detected by MS. The detailed schematic for the PC alkyne biotin structure and reaction procedure is shown in Supplementary Fig. 1.

We have tested different endoglycosidases for their specificity and selected three endoglycosidases for our method. To evaluate the specificity of the selected endoglycosidases, glycopeptides from MCF7 cells were incubated with Endo-H, Endo-S and Endo-F3, respectively. The released glycans were collected, labeled with procainamide through reductive amination and analyzed by nano LC-MS. The result showed that five high mannose and four hybrid N-glycans could be released by Endo-H (Supplementary Fig. 2a). The substrates for Endo-S were all bi-antennary complex glycans (Supplementary Fig. 2b). The EndoF3 released the non-fucosylated tri-antennary and core fucosylated bi/tri-antennary glycans (Supplementary Fig. 2c). However, bisected bi-antennary structures with/without core fucose were not substrates for Endo-F3 (Supplementary Fig. 3). None of these three endoglycosidases showed any activity towards the more complex tetra-antennary structures. The detailed information of endoglycosidase specificity is listed in Supplementary Table 1. Therefore, using the three 
endoglycosidases in our method, the quantitative assessment of six N-glycan types at glycopeptides was achieved and the sex types of $\mathrm{N}$-glycan were classified as following: non-fucosylated high mannose/hybrid, non-fucosylated bi-antennary, non-fucosylated tri-antennary, core fucosylated high mannose/hybrid, core fucosylated bi-antennary and core fucosylated bi/tri-antennary type glycans (Fig. 1b).

Our method also includes a novel enrichment strategy. Previously, GalT1 Y298L was used to label the O-linked $\beta$ - $N$-acetylglucosamine (GlcNAc) glycopeptide with GalNAz ${ }^{27}$. We discovered that the GalT1 Y298L could effectively label the N-GlcNAc (on average 95.3\%) as shown in Supplementary Fig. 4. After the N-linked glycopeptides were modified with GalNAz, and covalently linked with photocleavable biotin through click reaction, the unlabeled peptides and other reagents were removed by extensive washing with urea and organic buffer. The PC biotin alkyne was selected for our method development because ultraviolet irradiation is a milder condition to release the labeled glycopeptides, when compared with chemical methods that use strong reductive hydrazine or oxidizing regents ${ }^{28}$. Irradiation with $365 \mathrm{~nm}$ ultraviolet light efficiently recovered glycopeptides from the agarose streptavidin beads, with almost all glycopeptide released within 15 min (Supplementary Fig. 5).

\section{Validating the Glyco-TQ method on the standard glycoprotein IgG1 Fc}

We first tested our approach using an immunoglobulin G1 (IgG1), containing one fragment crystallizable region ( $\mathrm{Fc}$ fragments) and antigen-binding fragments ( $\mathrm{F}\left(\mathrm{ab}^{\prime}\right)_{2}$ fragment). It has one fixed N-glycosylated site at Asn297 of the Fc fragment while glycan occupancy on the $\mathrm{F}\left(\mathrm{ab} \mathrm{b}_{2}\right)_{2}$ fragments are reported to be near $20 \%{ }^{29}$. To obtain one standard $\mathrm{N}$-glycoprotein with one fixed glycosylated site, IgG1 from human serum was treated with IdeS protease, to generate a homogenous pool of $F\left(a b^{\prime}\right) 2$ and $F c / 2$ fragments and then the Fc fragments were enriched by protein A agarose chromatography (Fig. 2a). As proof of principle for our quantitative method, the proportion of each glycan type was investigated by both matrix-assisted laser desorption/ionization-MS (MALDI-MS) and our novel Glyco-TQ method. The $\mathrm{N}$-glycan spectrum of the Fe fragment was interrogated by MALDI-MS detection in Fig. $2 b$. The proportion of different types of $\mathrm{N}$-glycans was calculated through the peak intensity of each glycan structure based on the MALDI-MS spectrum. The detailed ratio information of each structure is shown in Supplementary Table 2 for the MALDI-MS detection. For Glyco-TQ method, glycopeptides with sequence EEQYN\#STYR were classified into six types based on their linked glycan structure: nonfucosylated high mannose/hybrid type, non-fucosylated bi-antennary type, non-fucosylated tri-antennary 
type, core fucosylated high mannose/hybrid type, core fucosylated bi-antennary type and core fucosylated bi/tri-antennary type glycan. The MS spectra of enriched glycopeptides and their corresponding extracted-ion chromatograms are shown in the Fig. 2c. The comparison of the proportion of glycan types based upon our Glyco-TQ method and MALDI-MS detection is shown in Fig. 2d. The high mannose and hybrid glycan type was not detected using either methods, showing the high specificity of Endo-H. As for the bi-antennary glycans released by Endo-S, the proportion of the core fucosylated bi-antennary glycan is $93.9 \%$ using our Glyco-TQ method and $90.5 \%$ using MALDI-MS detection; the proportion of the non-fucosylated bi-antennary is $6.1 \%$ using our Glyco-TQ method versus $9.5 \%$ using MALDI-MS detection. The proportion of the core fucosylated bi-antennary from our method is higher than using MALDI-MS detection (93.9\% versus $90.5 \%$ ), which may be due to their different ionization modes. The Endo-F3 did not release the core fucosylated bisecting bi-antennary glycans and nonfucosylated bi-antennary type glycans (showing only with minor activity when compared with triantennary glycans) (Supplementary Fig. 4$)^{30}$. Only 3.3\% of non-fucosylated glycans were released by the Endo-F3 in these experiments. In conclusion, our Glyco-TQ method quantitatively revealed glycan type and linkage of IgG1 Fc.

\section{Identification of N-glycopeptides from MCF7 cells}

Detection of endogenous and native N-linked GlcNAc glycopeptides. Similar to endoglycosidases, Endo- $\beta$-N-acetylglucosaminidase (ENGase) acts as a de-glycosylation enzyme for the misfolding proteins in the cytosol ${ }^{31}$. We first applied our enrichment method to enrich the endogenously and native existing N-linked GlcNAc glycopeptides from MCF7 total cell lysates in absence of added any endoglycosidase (Supplementary Fig. 6). Our enrichment strategy yielded 71 N-linked glycopeptides that mapped to consensus N-glycosylated sequences (Asn-X-Ser-Thr-Cys) and that were detected with modification of one N-linked GlcNAc residue (peptide-GlcNAc) (Supplementary Table 3). At the same time, we also detected 63 O-linked GlcNAc modified peptides, which are located in the nucleus and cytoplasm by gene ontology (GO) analysis (Supplementary Table 4). Caution should be taken when interpreting results since $\beta$-N-Acetylhexosaminidase couldn't fully remove some abundant O-linked GlcNAc modifications.

Detection of N-glycopeptides from MCF7 cells. To verify our enrichment strategy, we applied the three endoglycosidases $(\mathrm{H}, \mathrm{S}, \mathrm{F} 3)$ to help us enrich all the high mannose, hybrid, bi- and tri-antennary complex linked glycopeptides (Supplementary Fig. 7). To evaluate the reproducibility of our enrichment 
method, we performed three biological replicates with MCF-7 protein cell lysates and found $73 \%$ glycopeptides were identified in at least two replicates (Supplementary Fig. 8). We compared the glycopeptide and non-glycopeptide fractions in each parallel replicate, and showed that the specificity of our method is $55.4 \%$. Our performance was better than the specificity of previously boronic acid and ZIC-HILIC enrichment method ${ }^{8}$. After N-glycan was released by the endoglycosidase, the core fucose was retained on the core GlcNAc residue, which allowed us to simultaneously distinguish the nonfucosylated and core fucosylated peptides. In total, $1090 \mathrm{~N}$-glycopeptides were detected, including 916 non-fucosylated and 174 core fucosylated glycopeptide, corresponding to 504 glycoproteins (Supplementary Table 5). There were 116 glycopeptides with co-occurrence of the non-fucosylated and core fucosylated glycopeptides (Supplementary Fig. 9). As well, 58 glycopeptides were detected with only core fucosylated type glycans. Amino acid frequencies of sequences surrounding the $\mathrm{N}$-glycosylated site are shown in Supplementary Fig. 10 for both the canonical and atypical N-linked glycopeptides. The above was carried out using the stepped fragmentation strategy. When the normalized collision energy (NCE) was set to 15 , the fuc-GlcNAc linkage was prone to cleavage, which resulted in the parent ion and fucose neutral loss-ion as the highest peaks (Supplementary Fig. 11). For example, one MS/MS spectrum of atypical motif glycopeptide with sequence ISVN\#NVLPVFDNLMQQK was identified with one core fucose as shown in the Fig. 3. In addition, the glycopeptides with common glycan tag (GlcNAcGalNAzPCt) allowed help us to identify the glycopeptides with two glycosylated sites (Supplementary Fig. 12), overcoming significant challenges posed for their identification when using the intact glycopeptide method (5-7 KDa) ${ }^{32}$.

\section{Applying the Glyco-TQ method to analyze glycopeptides of MCF7 cell line}

Quantitative micro-heterogeneity of glycopeptides and glycoproteins. The chemoenzymatic strategy allowed us to quantify the $\mathrm{N}$-linked glycan dynamics on specific glycopeptide. After the peptides were treated with Endo-H, S and F3, we combined and enriched the glycopeptides by affinity chromatography (Fig. 1a). Relative quantitation of isotope peaks was calculated using the intensity of each peak area, which represented the proportion of each glycan type on the glycopeptide. First, we evaluated whether the different endoglycosidases treatment introduced biases during the glycan releasing steps. As show in Supplementary Fig. 13, high values from correlation coefficients (the value of Pearson's correlation coefficient $r>0.96$ ) were observed between the different endoglycosidase treatment after isotope labeling. Thus, we concluded that the endoglycosidase did not exhibit off-target 
protease activity and so did not introduce sample bias during glycan releasing steps. In total, we quantitatively detected 698 peptides that mapped to 378 proteins. All the glycopeptides and the relative ratio of each glycan type are shown in Supplementary Table 6. Almost all the non-fucosylated glycopeptides (>97\%) linked with the high-mannose or hybrid glycans (Fig. 4a), while only 2 glycopeptides were linked with core fucosylated hybrid glycan and more than $97 \%$ core fucosylated peptides were linked with bi- and tri-antennary complex glycans (Fig. 4b). The quantitative results exhibit distinct expression profiles for six glycan types on the glycopeptide as shown in the Fig. 4c, highlighting the abundant expression of non-fucosylated high-mannose and hybrid N-glycan. We use the heatmap to show the quantitative micro-heterogeneity of glycopeptide, and relative distribution of glycan type present on the particular glycosylated site (Fig. 4d-f). For the non-fucosylated glycopeptide, 143 glycopeptides were only linked with high-mannose and hybrid type glycan (Fig, 4d). Non-fucosylated glycopeptides $(86.3 \%)$ were linked with high-mannose and hybrid type glycan, of which their proportion is larger than 50\% (Supplementary Table 6 and Fig, 4d). Consistent with previous report, our results showed that N-glycans from the MCF7 cell line were predominant of non-fucosylated highmannose/hybrid type glycans ${ }^{33}$. That is expected as all of the glycoproteins were first linked with high mannose type glycan during the biosynthesis ${ }^{34}$. As for the quantitative micro-heterogeneity on the protein level, we studied internal connection of different glycopeptides from the same glycoprotein (Fig. 4g-i). The distance between the connected dots represents the glycan type expressing divergence of glycopeptides from the same protein. Some proteins with more than one glycosylated site, such as hemicentin-1, membralin and deoxyribonuclease-2-alpha, were detected only linked with the nonfucosylated high-mannose and hybrid type glycans, released by Endo-H. However, the majority of detected proteins with multiple glycosylated sites have differential N-glycan type profiles at each site for both the non-fucosylated (Fig. 4g, h) and fucosylated glycoproteins (Fig, 4i). Due to the massive expression of non-fucosylated high-mannose and hybrid type, distribution of non-fucosylated proteins was constricted to a small region (Fig. 4g, h), while the fucosylated glycoproteins were more widely distributed, based upon the quantitative information of six N-glycan types (Fig. 4i). We also compared the location of non-fucosylated and fucosylated glycoproteins and found that more than $60 \%$ of fucosylated proteins were located in the extracellular exosome while for non-fucosylated counterparts, it roles in cell adhesion and molecular trafficking. 

reported to be related with cancers. Mannose-6-phosphate receptor (M6PR), for example, can regulate cell growth and motility, and it functions as a breast cancer suppressor ${ }^{35}$. We detected seven Nglycopeptides from that protein, which exhibited diverse structures on each site (Fig. 5). The glycopeptides with sequence MN\#FTGGDTCHK,

TN\#ITLVCKPGDLESAPVLR, N\#GSSIVDLSPLIHR had similar glycan type profiles, only expressing the non-fucosylated glycan on those sites. In addition, the ratio of non-fucosylated high mannose/hybrid type glycans are more than $77 \%$ in those three peptides. The glycopeptides with sequence MDGCTLTDEQLLYSFN\#LSSLSTSTFK only expressed the core fucosylated complex glycan, which could only be released Endo-F3, not Endo$\mathrm{S}$, indicating that the glycan structures were all tri-antennary core fucosylated $\mathrm{N}$-glycans. The glycopeptide with sequence TGPVVEDSGSLLLEYVN\#GSACTTSDGR had the most complex glycan profile, expressing both high mannose (14.9\%) and fucosylated complex glycans (85.1\%). The M6PR showed heterogeneity of glycan structure and distinctive glycan profiles on each of its glycopeptides. Through investigating the proportion of glycan types at each site, we could get a better understanding of the expression of this glycoprotein and its glycoprotein variants, which will promote our understanding of glycoprotein function in cancer.

\section{DISCUSSION}

The previously quantitative reports of glycoproteome generally focused on the difference of one specific structure at glycopeptides between samples. The distinctive characteristic of our research is that we could provide the relative proportion of N-glycan types on each glycopeptide, which indicate the activity and aberration expression of related glycotransferases. That novel quantitative strategy provides broad information on each glycosylated site, such as the ratio of high-mannose and core fucosylated glycan, the construction of bi/tri-antennary about the complex glycans. The specific types of glycan contribute important property of glycoproteins. For example, an antibody drug linked with high-mannose type glycan showed decreased complement activity and thermal stability ${ }^{36,37}$. However, it is challenging to detect the low abundance N-glycans, including the high-mannose and hybrid structures ${ }^{35}$. We can directly provide the proportion of high-mannose and hybrid structure with help of glycol-TQ strategy, which has potential to become the routine analysis strategy for pharmaceuticals. In addition, the increasing expression of the core fucosylated type on specific glycoprotein was as potential biomarkers 
in samples from patients with hepatocellular carcinoma (HCC) than chronic hepatitis and liver cirrhosis, and so has been approved for the early detection of $\mathrm{HCC}^{19}$. Taking advantage of our method, we can provide not only the expression level of fucosylated AFP L3, but also the relative ratio between nonfucosylated and core fucosylated AFP L3. Combining both level of information maybe a more sensitive and specific strategy to investigate the fucosylated biomarker. Additionally, the proteomic method could simultaneously detect multiple potential glycoproteins from single analysis. In the area of anti-glycan drug development, the N-linked glycan of HIV-1 Env is the target for broadly neutralizing antibodies, therefore, routine analysis of glycan structure supports rational design and development of vaccine immunogens ${ }^{22}$. Our Glyco-TQ method makes it possible to quantitatively detect glycan types on each site of human immunodeficiency virus (HIV) envelope glycoprotein (Env) trimer without extensive purification. Therefore, our Glyco-TQ method has great potential in the area of biomarker research, antiglycan drug development and fundamental biological research.

Our strategy used relatively mild-conditions and achieved high specificity through the biotin-avidin affinity chromatography. The high specificity was contributed: the strength of the biotin-avidin binding that allows us to extensively wash to remove non-specific peptides. As well, the photocleavable tag $(\mathrm{PCt})$ added to the GalNAz has one amide group, all the glycopeptides should exist in charge state of $3+$ or more. The 2+ non-specific peptides wouldn't be detected in the MS analysis, as we set the most abundant $3+$ to $6+$ peptides for the MS/MS analysis in the data-dependent acquisition mode. Unlike other modifications, such as phosphorylation and acetylation, the diverse glycan structures on glycoproteins make their analyses extraordinarily challenging by MS. In order to comprehensively investigate glycosylation sites, introducing a common tag could provide convenience for glycopeptide confirmation. PNGase $\mathrm{F}$ is the most common enzyme used to hydrolyze the glycosylamine linkage between N-glycans and asparagine, introducing a universal mass tag (0.98 Da shift) as the asparagine residue is converted to aspartic acid. However, spontaneous non-enzymatic deamidation of asparagine residues significantly affect the accuracy of the N-linked glycosylation site determination ${ }^{38}$. In our enrichment strategy, all processed glycopeptides contained one unique glycan residue (GlcNAc-GalNAzPCt, 502.2 Da or FucGlcNAc-GalNAzPCt, $648.2 \mathrm{Da}$ ). That common tag not only reduced the false identification of glycopeptides, but also distinguished the non-fucosylated and core fucosylated glycopeptide. For example, the peptide labeled with Fuc-GlcNAc-GalNAzPCt, $648.2 \mathrm{Da}$ was only mapped to the N- 

the HCD dissociation mode couldn't directly tell the glycosite of glycopeptides ${ }^{39}$. The glycan-peptide linkage is more labile than the amino acid linkages, which leads to the core GlcNAc residue first release from peptide rather than peptide dissociation under high HCD energy. Although the N-glycopeptide canonical sequon $(\mathrm{N}-\mathrm{X}-\mathrm{T} / \mathrm{S})$ would help us overcome most of interference of O-linked GlcNAc modifications, that problem could be further resolved by using the more advanced EThcD dissociation strategy, which would be able to locate the modified site from the MS/MS spectrum. glycopeptide, while, the intact glycopeptides generally have lower ionization efficiency, when compared with their peptide counterparts ${ }^{40}$. Heterogeneity of the glycan structures from the intact glycopeptide produces a number of sub-stoichiometric modifications, splitting MS signals of the same glycopeptide into a broad spectrum of ion species ${ }^{41}$. Thus, the intact glycopeptide method qualitatively detects the most abundance structures for a glycopeptide, while the information of minor glycan structure on the same glycopeptide will be ignored during the MS detection. On the contrary, using our novel Glyco-TQ methods, six type structures based on the N-glycan linkage and terminal from the glycopeptide were quantified by the intensity of MS signal. Therefore, site-specific intact glycopeptide detection and our Glyco-TQ method could become complementary strategies and come together to provide both qualitative and quantitative information, facilitating further understanding of the structure and function of glycoproteins. Finally, biologists could use our strategy to directly labeling their N-glycoproteins of interest. The GalNAz labeled glycoprotein could then be modified by PEG mass tag, resolved by SDSPAGE and visualized through immunoblotting with antibodies ${ }^{42}$. That strategy would permit rapid quantitation of $\mathrm{N}$-glycosylation levels of particular protein without need for purification or expensive instruments, such as MS.

In conclusion, we provide a chemoenzymatic method to quantify the glycan type on the glycoproteins. All the procedures were with mild-conditions and result showed high specificity of enrichment through affinity chromatography. We provide a new quantitative strategy based the glycan type, which allows us assess the micro-heterogeneity of glycoproteins. The Glyco-TQ method has potential to be used in broad areas, such as biomarker research, pharmaceuticals, and fundamental biological research. 


\section{Figure Legends}

349 Fig. 1 The enrichment and quantitative strategy for N-glycoproteomics. a The workflow for the N-

350 glycopeptide enrichment and quantitation. b Six N-glycan types of glycan structures linked on the 351 glycopeptide: non-fucosylated high mannose/hybrid type, non-fucosylated bi-antennary, non-fucosylated tri-antennary, core fucosylated high mannose/hybrid type, core fucosylated bi-antennary, core fucosylated bi/tri-antennary type.

Fig. 2 Quantitative analysis the standard glycoprotein IgG1 Fc using the Glyco-TQ method. a Purification of Fc fragment from human serum IgG1. b MALDI-MS detection the glycan profile of Fc fragments. c Quantitative investigation the proportion of Fc fragment glycopeptide, the MS profile of glycopeptide (up) and the corresponding extracted-ion chromatogram of glycopeptide. d Quantitative comparison of MALDI-MS method and Glyco-TQ method basing on the glycan type.

Fig. 3 MS/MS spectrum of atypical motif glycopeptide with sequence ISVN\#NVLPVFDNLMQQK. * represents the $\mathrm{b}$ or $\mathrm{y}$ ions losing the glycan common tag. The neutral loss of fucose was shown between the parent ion at $\mathrm{m} / \mathrm{z} 2608.2$ and the fucose neutral loss-ion at $\mathrm{m} / \mathrm{z} 2462.2$. The oxonium ions from the common glycan tag was set as diagnostic ion peak, representing the fragment of GlcNAz at m/z 204.0, GalNAzCA at m/z 300.1 and GlcNAc-GalNAzPCt at m/z 503.2. The mass shift between b7 and b7* (* represent losing the common tag modification) is $618.3 \mathrm{Da}$, which exactly the mass of the common glycan tag (Fuc-GlcNAc-GalNAzPCt).

Fig. 4 Quantitative detection MCF 7 cell derived glycopeptides by the Glyco-TQ method. a Detection of the non-fucosylated glycopeptide based on the glycan type. b Detection of the core fucosylated glycopeptide based on the glycan type. c The different N-glycan type ratio of glycopepide. d Quantitative detection of non-fucosylated glycopeptide. e Quantitative detection of both non-fucosylated and core fucosylated glycopeptide. f Quantitative detection of core fucosylated glycopeptide. Each row indicates one specific glycopeptide, and each column indicates the one type of glycan structure. The relative intensity of each glycan type on the glycopeptide was used for two-dimensional hierarchical clustering analysis. $\mathbf{g}$ The glycoprotein with two non-fucosylated glycosylation sites. $\mathbf{h}$ The glycoprotein with three or more non-fucosylated glycosylation sites. i The glycoprotein with two or more core fucosylated glycosylation sites, as the fucosylated glycopeptide includes non-fucosylated section and core fucosylaeted section: - represents non-fucosylated section of core fucosylated glycopeptides, represents fucosylated section of core fucosylated glycopeptides. The distribution of each glycopeptide 
378 on the Fig. $4 \mathrm{~g}-\mathrm{i}$ based on the relation ratio (\%) of each $\mathrm{N}$-glycan type. The glycopeptides from the same

379 glycoprotein was linked together.

380 Fig. 5 Quantitative analysis of glycosylation of mannose-6-phosphate receptor. \# represents the 381 glycosylated site.

382

383

384

385

386

387

388

389

390

391

392

393

394

395

396 
bioRxiv preprint doi: https://doi.org/10.1101/803494; this version posted October 15, 2019. The copyright holder for this preprint (which was not certified by peer review) is the author/funder. All rights reserved. No reuse allowed without permission.

Fig. 1

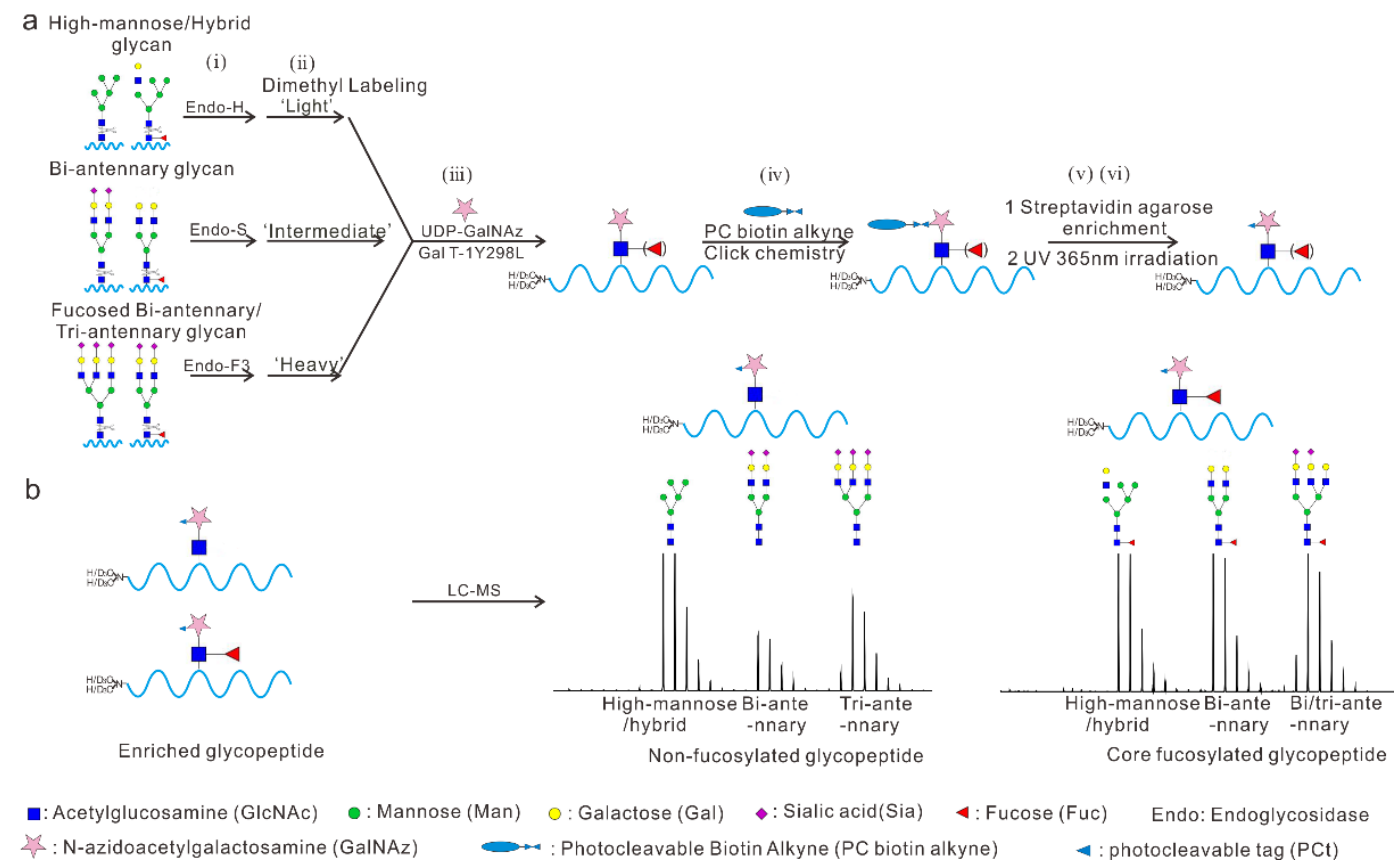

398

399

400

401

402

403

404

405

406 
Fig. 2

a

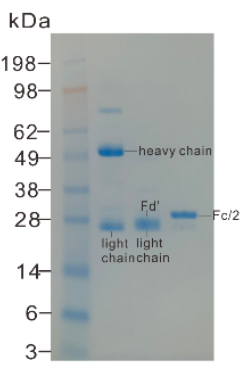

C

407
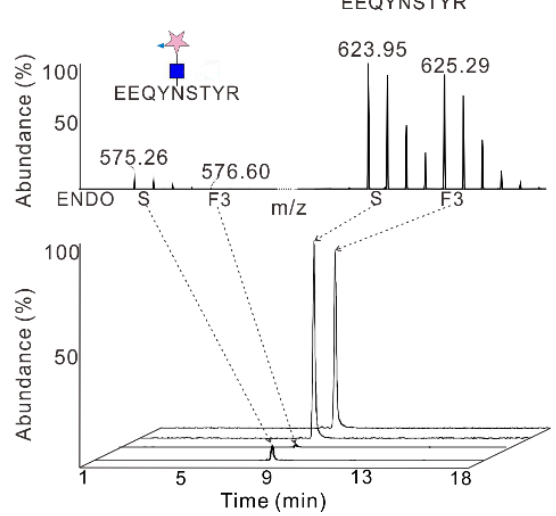

b

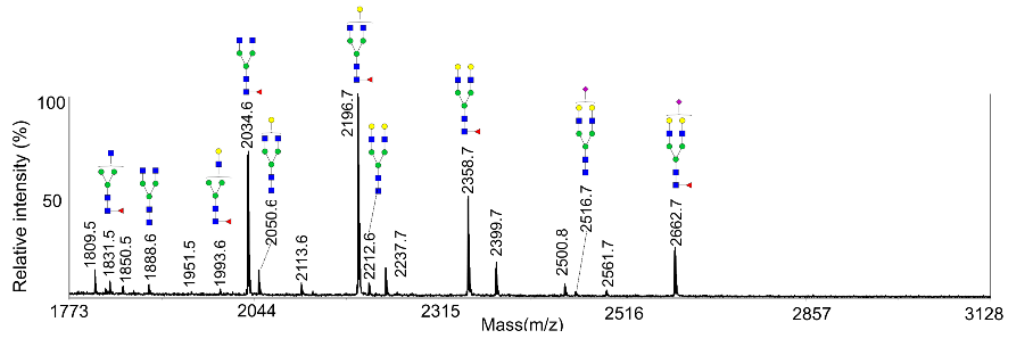

d

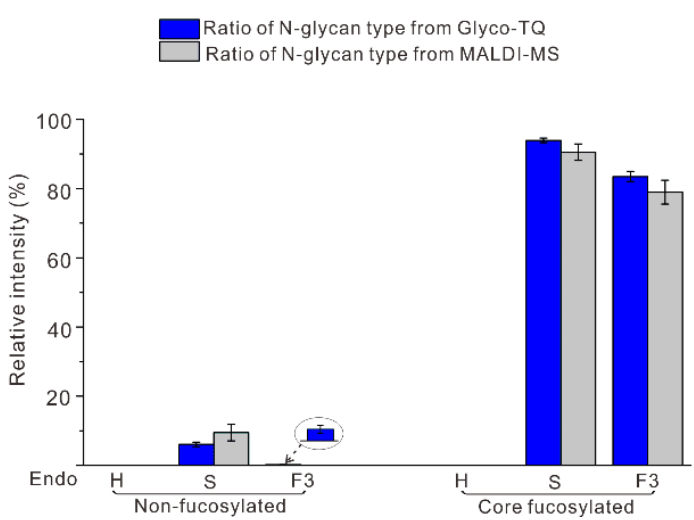

408

409 
bioRxiv preprint doi: https://doi.org/10.1101/803494; this version posted October 15, 2019. The copyright holder for this preprint (which was not certified by peer review) is the author/funder. All rights reserved. No reuse allowed without permission.

Fig. 3

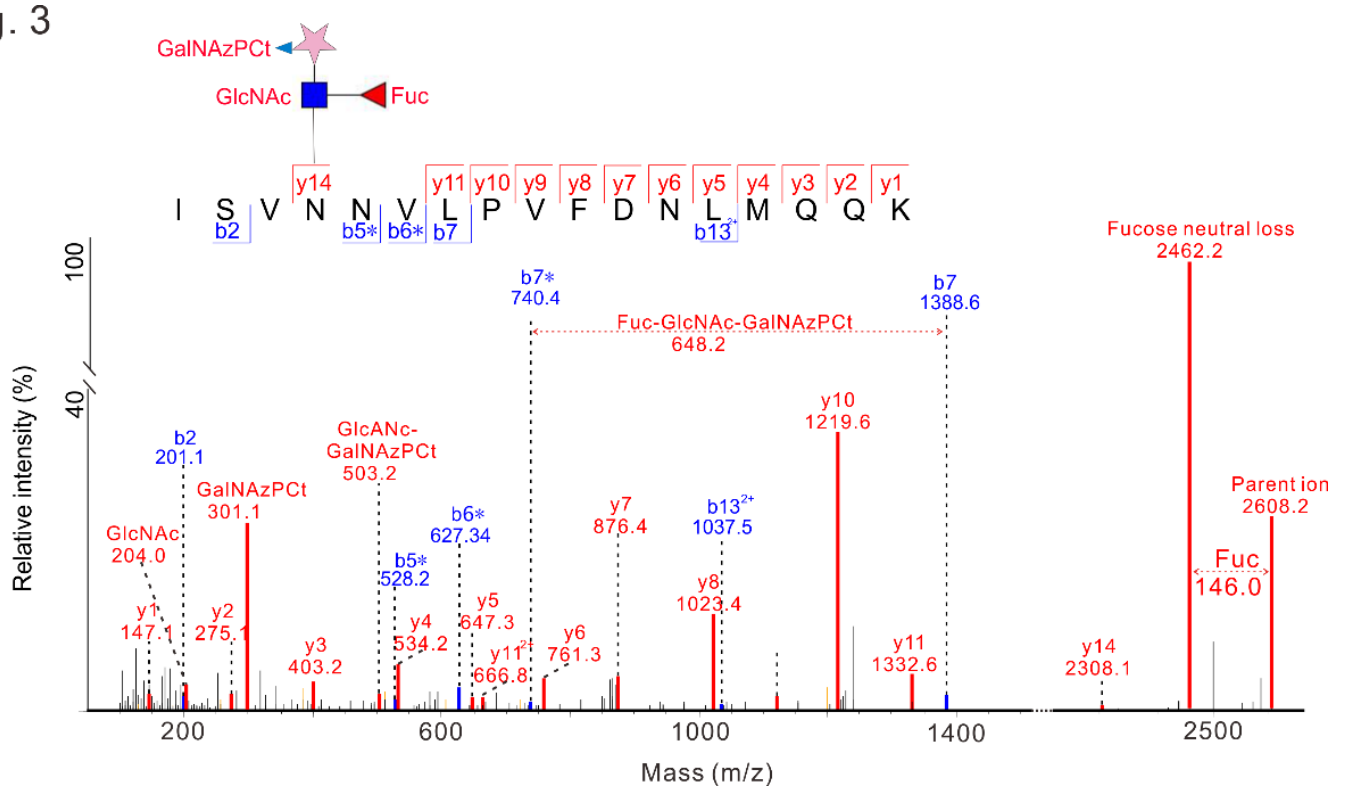

415 
bioRxiv preprint doi: https://doi.org/10.1101/803494; this version posted October 15, 2019. The copyright holder for this preprint (which was not certified by peer review) is the author/funder. All rights reserved. No reuse allowed without permission.

Fig. 4

a

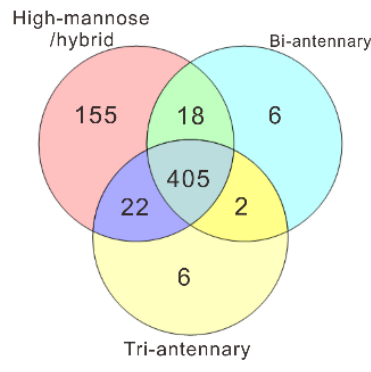

Non-fucosylated glycopeptide b

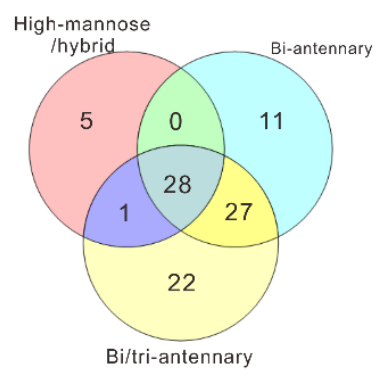

Core fucosylated glycopeptide

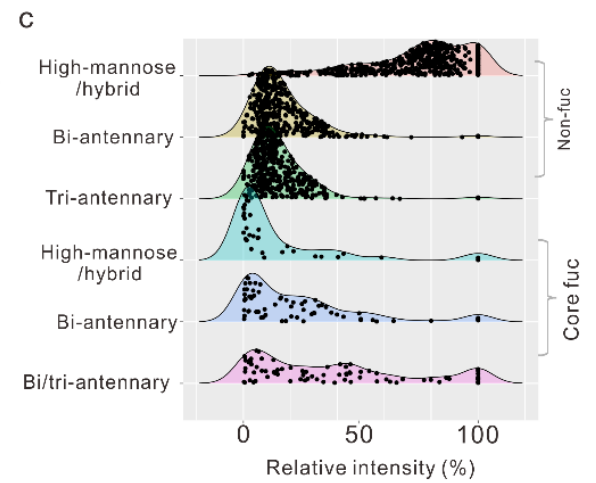

e

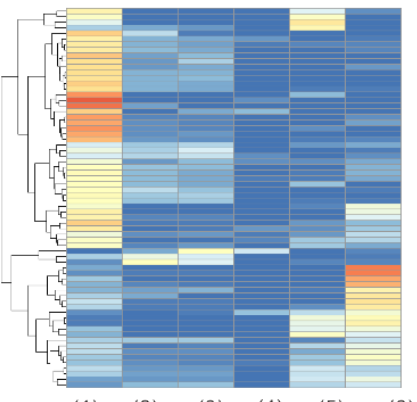

$\begin{array}{lllll}(1) & \text { (2) (3) (4) (5) (6) }\end{array}$

Non-/core fucosylated glycopeptide h

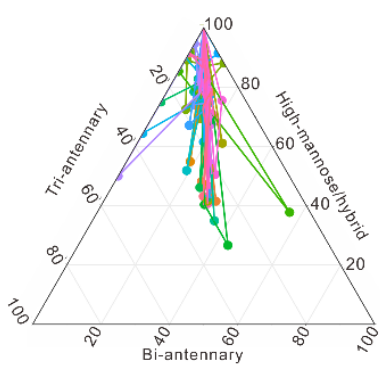

Non-fucosylated glycoproteins

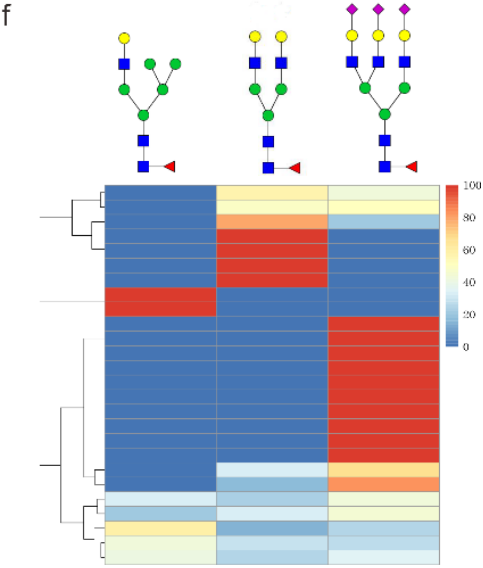

(4) High-man- (5) Bi-ante (6) Bi/Tri-an
nose/hybrid Core fucosylated glycopeptide

i

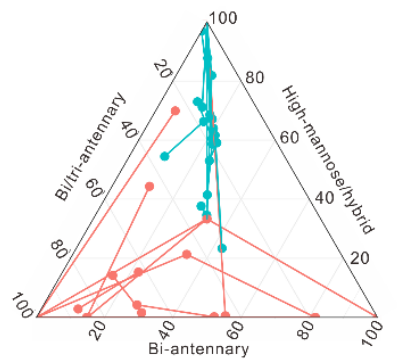

Core fucosylated glycoproteins 
Fig. 5

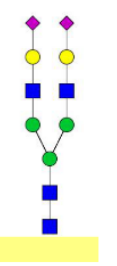

High-mannose /hybrid

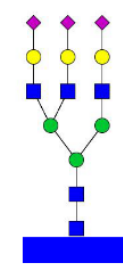

Tri-ante -nnary

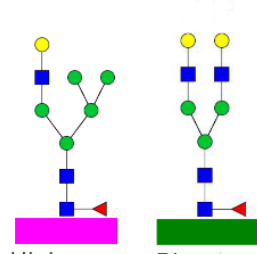

High-man- Bi-anten nose/hybrid -nary

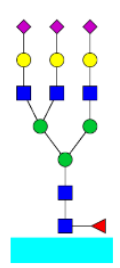

Bi/Tri-ante -nnary

mannose-6 phosphate receptor

TGPVVEDSGSLLLEYVN\#GSACTTSDGR

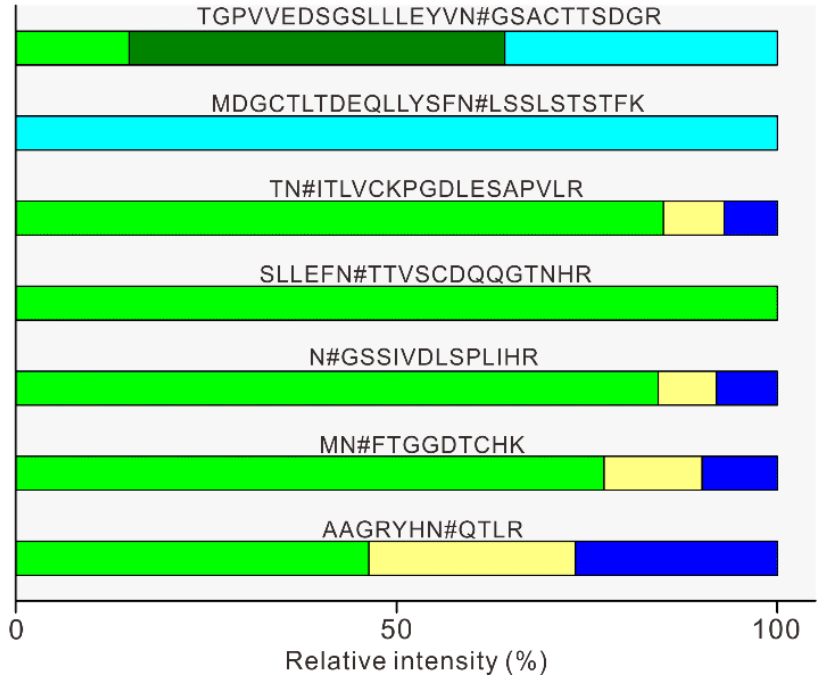

426 


\section{METHODS}

\section{Materials}

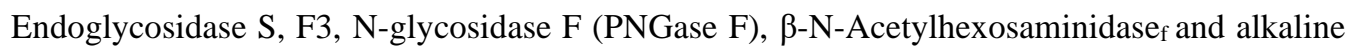
phosphatase were from New England Biolabs. Mutant B1-4-Galactosyltransferase (Gal-T1 Y289L)) and High Capacity Streptavidin Agarose were obtained from Thermo Scientific. IdeS protease was purchased from Promega. UDP-GalNAz was from chemily Glycoscience. The immunoglobulin G1 (IgG1) from normal human plasma was obtained from Athens Research \& Technology. 2-(4((bis((1-(tert-butyl)-1H-1,2,3-triazol-4-yl) methyl) amino) methyl)-1H-1,2,3-triazol-1-yl) acetic acid (BTTAA), photocleavable biotin alkyne (PC biotin alkyne) were purchased from Click Chemistry Tools. EDTA-free protease inhibitor cocktail was obtained from Roche Diagnostics. RapiGest SF surfactant and the Sep-Pak tC18 cartridge was obtained from Waters. All other chemical materials, if not special highlighted, was obtained from Millipore Sigma.

\section{Preparing the standard glycoprotein}

Immunoglobulin G1 $(100 \mu \mathrm{g})$ was treated with 1000 units IdeS protease for 3 hours in 1X phosphate buffered solution (PBS, pH 7.6). $200 \mu \mathrm{L}$ of immobilized Protein A resin slurry (50\% w/v) was added to the reaction buffer, and incubated with gentle mixing for 2 hours at room temperature. Then the Protein A resin slurry were transferred into centrifuge columns and Protein A resin was washed with 1 XPBS three times to remove unbound $\mathrm{F}\left(\mathrm{ab}^{\prime}\right)_{2}$ fragments (fragment antigen-binding). The Fc fragments (fragment crystallizable region) of immunoglobulin G1 (IgG 1) were eluted with $100 \mathrm{mmol} / \mathrm{L}$ glycine buffer, $\mathrm{pH}$ 2-3. The Fc fragments were immediately neutralized with $1 \mathrm{M}$ Tris- $\mathrm{HCl}$ buffer and stored in $-80^{\circ} \mathrm{C}$ for further use.

\section{Cell culture, protein extraction and protein digestion}

The MCF-7 cell line was obtained from American Type Culture Collection (ATCC). MCF-7 cells were maintained in advanced MEM media (Gibco) with 10\% (v/v) FBS, 1X GlutaMAX (Gibco), and $2.8 \mu \mathrm{g} / \mathrm{mL}$ Gentamicin (Gibco). The cells were cultured at $37{ }^{\circ} \mathrm{C}$ and $5 \% \mathrm{CO}_{2}$. Once the cells reached $80 \%$ confluency, cells were harvested in the ice-cold RIPA buffer (50 mM HEPES $\mathrm{pH} 7.6,150 \mathrm{mM} \mathrm{NaCl}, 1 \% \mathrm{NP}-40,1 \%$ sodium deoxycholate, $0.1 \%$ sodium dodecyl sulfate (SDS) and protease inhibitor cocktail (CompleteMini, Roche)) by scraping. Cell lysates were subjected to ultrasonicate (10 s process with $10 \mathrm{~s}$ interval for $1 \mathrm{~min}$ ) on ice using a Q125 Sonicator with 50\% amplitude. The cell debris was removed through centrifugation at $16000 \mathrm{~g}, 4^{\circ} \mathrm{C}, 10 \mathrm{~min}$. The protein 
472 in the supernatant was precipitated using 6-fold volume ice cold acetone overnight at $-20{ }^{\circ} \mathrm{C}$.

473 Protein was pelleted by centrifugation at $16000 \mathrm{~g}, 4^{\circ} \mathrm{C}, 10 \mathrm{~min}$ and washed with the ice-cold acetone

474 two times. For the in-solution trypsin digestion, the procedure was performed as the previously report $^{43}$. Briefly, the resulting protein was dissolved in $50 \mathrm{mM}$ ammonium bicarbonate and $8 \mathrm{M}$ urea solution, reduced in $5 \mathrm{mM}$ dithiothreitol (DTT) $\left(56^{\circ} \mathrm{C}, 30 \mathrm{~min}\right)$, and alkylated by with $10 \mathrm{mM}$ iodoacetamide $\left(25^{\circ} \mathrm{C}, 40 \mathrm{~min}\right.$ in the dark). Cell proteins were digested with the protein: trypsin (Worthington Biochemical Corp) at ratio, 50:1, in $50 \mathrm{mM}$ ammonium bicarbonate, $1 \mathrm{M}$ urea solution $\mathrm{pH} 7.8$ at $37^{\circ} \mathrm{C}$ for 20 hours. After the digest, the solution was acidified ( $\mathrm{pH} \mathrm{2-3)} \mathrm{by} 0.5 \%$ formic acid and centrifuged to remove the debris, the supernatant was collected and peptide was desalted by Sep-Pak tC18 cartridge (Waters). The peptide elution was dried by SpeedVac concentrator (Thermo Scientific).

\section{Endoglycosidases digestion and dimethyl labeling}

To leave one $\mathrm{N}$-acetylglucosamine for high mannose linked $\mathrm{N}$-peptide, peptide from $1 \mathrm{mg}$ protein was parallelly digested with $0.05 \mathrm{U}$ Endo-H (sigma) in the $50 \mathrm{mM}$ sodium acetate buffer, $\mathrm{pH} 6 ; 2000$ units Endo-S (NEB) for biantennary N-glycan in $50 \mathrm{mM}$ sodium acetate, $5 \mathrm{mM}$ calcium chloride buffer $\mathrm{pH}$ 5.5; 100 units Endo-F3 (NEB) in $50 \mathrm{mM}$ sodium acetate, $\mathrm{pH} 4.5$, for 24 hours respectively. After the endoglycosidase were denatured at $95^{\circ} \mathrm{C}, 5 \mathrm{~min}, \beta-\mathrm{N}$-acetylhexosaminidase $\mathrm{f}_{\mathrm{f}}$ was added to remove OGlcNAc for another 8 hours. After desalted by Sep-Pak tC18 cartridge, the peptide was adjusted to $\mathrm{pH} 6$ with HEPES buffer. For isotope dimethyl labeling, the peptides were treated with $10 \mu \mathrm{L} 20 \% \quad(\mathrm{v} / \mathrm{v})$ $\mathrm{CH}_{2} \mathrm{O}, 15 \mu \mathrm{L} 3 \mathrm{M} \mathrm{NaBH}_{3} \mathrm{CN}$ for the Endo-H treated sample; $10 \mu \mathrm{L} 20 \%$ (v/v) $\mathrm{CD}_{2} \mathrm{O}, 10 \mu \mathrm{L} 3 \mathrm{M} \mathrm{NaBH}_{3} \mathrm{CN}$ for the Endo-S treated sample; $15 \mu \mathrm{L} \mathrm{20 \% (v/v)} \mathrm{CD}_{2} \mathrm{O}, 15 \mu \mathrm{L} 3 \mathrm{M} \mathrm{NaBD} \mathrm{CN}_{3}$ for the Endo-F3 treated sample, at $25^{\circ} \mathrm{C}$ for 45 min with mixing. The reaction was quenched by adding $10 \mu \mathrm{L} 20 \%$ (v/v) ammonia solution, combined, purified by Sep-Pak cartridge, and dried by Speedvac.

\section{Glycopeptide enrichment}

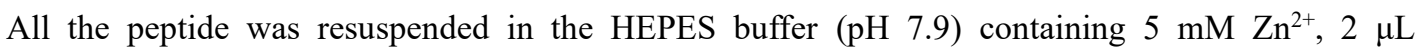
phosphatase, $10 \mu \mathrm{L}$ GalT1 T298L/1 mg peptide, and $25 \mu \mathrm{g}$ UDP-GalNAz/1 mg peptide, incubated in 4 ${ }^{\circ} \mathrm{C}$ for 24 hours. Excess UDP-GalNAz was remove by Sap-pak C18 cartridge. The peptide was dried in the speedVac and resuspended the PBS buffer. The Copper(I)-catalyzed Azide-Alkyne Cycloaddition (CuAAC) reaction reagents $(25 \mathrm{nmol} \mathrm{PC}$ biotin alkyne, $300 \mu \mathrm{M} \mathrm{CuSO} 4,600 \mu \mathrm{M}$ BTTAA, $1.50 \mathrm{mM}$ sodium ascorbate) was mixed with the GalNAz labeled peptide and the reaction was incubated for $3 \mathrm{~h}$ at 

$20 \%(\mathrm{v} / \mathrm{v})$ acetonitrile $(\mathrm{ACN})$ ten times. The beads were then resuspended in $50 \%(\mathrm{v} / \mathrm{v}) \mathrm{ACN}$, transferred transilluminator, LM-20E) for $30 \mathrm{~min}$ at $4{ }^{\circ} \mathrm{C}$ with gentle mixing. The supernatant from each fraction was collected, lyophilized, and stored at $-20^{\circ} \mathrm{C}$.

\section{Mass spectrometry analysis}

Glycopeptides were analyzed by an Eksigent nanoLC liquid chromatograph that was connected inline with an Q Exactive HF-X MS. The separation of peptides was performed on an analytical column $(75 \mu \mathrm{m} \times 50 \mathrm{~cm})$ packed with reverse phase beads $(1.9 \mu \mathrm{m} ; 120-\AA$ pore size; Dr. Maisch $\mathrm{GmbH})$ with 2hour gradient from 5 to $35 \%$ acetonitrile (v/v) at a flow rate of $200 \mathrm{nl} / \mathrm{min}$. The full scan mass spectrums were acquired over range $300-1800(\mathrm{~m} / \mathrm{z})$ with the mass resolution setting 70000 at $\mathrm{m} / \mathrm{z} 400$. Maximum injection time $100 \mathrm{~ms}$; AGC target value 1e6. The 12 most intense ions were selected for tandem mass spectrometry detection with the following parameters: collision energy, 30\%; exclusion ions charge 1, 2, 7, 8, >8; resolution 17500, AGC target 1e5; maximum injection time $120 \mathrm{~ms}$.

\section{Data analysis}

The raw data were processed using the MaxQuant software and searched against with UniProt human database containing all proteins in the UniProt Human (Homo sapiens) database (20190802). The general parameters were performed during the search: $10 \mathrm{ppm}$ precursor mass tolerances; digested with trypsin; two max missed cleavages; fixed modifications: carbamidomethylation of cysteine (+57.0214); variable modifications: oxidation of methionine $(+15.9949)$. The common tag was also performed as variable modifications: modified amino acid, asparagine (N); composition $\mathrm{H}_{30} \mathrm{C}_{19} \mathrm{~N}_{6} \mathrm{O}_{10}$, GlcNAc-GalNAzphotocleavable tag (GlcNAc-GalNAzPCt), 502.2023 Da; neutral losses, GlcNAc-GalNAzPCt, GalNAzPCt $\mathrm{H}_{17} \mathrm{C}_{11} \mathrm{~N}_{5} \mathrm{O}_{5}$; diagnostic peaks, GalNAzPCt $\mathrm{H}_{17} \mathrm{C}_{11} \mathrm{~N}_{5} \mathrm{O}_{5}$, GlcNAc $\mathrm{H}_{13} \mathrm{C}_{8} \mathrm{NO}_{5}$, GlcNAcGalNAzPCt. If the N-glycopeptide was modified with core fucose, fucosylated linked was performed as

527 variable modifications as following: modified amino acid, asparagine $(\mathrm{N})$; composition $\mathrm{H}_{40} \mathrm{C}_{25} \mathrm{~N}_{6} \mathrm{O}_{14}$, 


\section{Acknowledgements}

533 This work was supported by the Government of Canada through Genome Canada and the Ontario

534 Genomics Institute (OGI-114), CIHR grant (ECD-144627), the Natural Sciences and Engineering 535 Research Council of Canada (NSERC, grant no. 210034), the Ontario Ministry of Economic 536 Development and Innovation (REG1-4450) and The University of Ottawa. DF acknowledges a 537 Distinguished Research Chair from the University of Ottawa.

\section{Authors' contributions}

539 D.F., J.L, and H.L. designed the study. H.L., L.L, K.C and K.W performed the experiments and data analysis. R.C and S.T involved in discussion of the study design. D.F., J.L, H.L., X.Z, Z.N and J.M. wrote the manuscript. All authors participated in the data interpretation, discussion and edits of the manuscript.

\section{Competing interests}

543 D.F. was co-founded Biotagenics and MedBiome, clinical microbiomics companies. The remaining 544 authors declare no competing interests. 


\section{REFERENCE}

(1) Ohtsubo, K.; Marth, J. D. Glycosylation in cellular mechanisms of health and disease. Cell 2006, $126(5), 855$.

(2) Stanley, P.; Taniguchi, N.; Aebi, M. In Essentials of Glycobiology; rd;Varki, A.;Cummings, R. D.;Esko, J. D.;Stanley, P.;Hart, G. W.;Aebi, M.;Darvill, A. G.;Kinoshita, T.;Packer, N. H.et al., Eds. Cold Spring Harbor (NY), 2015, DOI:10.1101/glycobiology.3e.009 10.1101/glycobiology.3e.009.

(3) Hebert, D. N.; Lamriben, L.; Powers, E. T.; Kelly, J. W. The intrinsic and extrinsic effects of Nlinked glycans on glycoproteostasis. Nature chemical biology 2014, 10 (11), 902.

(4) Gagneux, P.; Varki, A. Evolutionary considerations in relating oligosaccharide diversity to biological function. Glycobiology 1999, 9 (8), 747.

(5) Zielinska, D. F.; Gnad, F.; Wisniewski, J. R.; Mann, M. Precision mapping of an in vivo Nglycoproteome reveals rigid topological and sequence constraints. Cell 2010, 141 (5), 897.

(6) Wollscheid, B.; Bausch-Fluck, D.; Henderson, C.; O'Brien, R.; Bibel, M.; Schiess, R.; Aebersold, R.; Watts, J. D. Mass-spectrometric identification and relative quantification of $\mathrm{N}$-linked cell surface glycoproteins. Nature biotechnology 2009, 27 (4), 378.

(7) Zhang, H.; Li, X. J.; Martin, D. B.; Aebersold, R. Identification and quantification of N-linked glycoproteins using hydrazide chemistry, stable isotope labeling and mass spectrometry. Nature biotechnology 2003, 21 (6), 660.

(8) Xiao, H.; Chen, W.; Smeekens, J. M.; Wu, R. An enrichment method based on synergistic and reversible covalent interactions for large-scale analysis of glycoproteins. Nature communications 2018, 9 (1), 1692.

(9) Mysling, S.; Palmisano, G.; Hojrup, P.; Thaysen-Andersen, M. Utilizing ion-pairing hydrophilic interaction chromatography solid phase extraction for efficient glycopeptide enrichment in glycoproteomics. Analytical chemistry 2010, 82 (13), 5598.

(10) Woo, C. M.; lavarone, A. T.; Spiciarich, D. R.; Palaniappan, K. K.; Bertozzi, C. R. Isotope-targeted glycoproteomics (IsoTaG): a mass-independent platform for intact $\mathrm{N}$ - and O-glycopeptide discovery and analysis. Nature methods 2015, 12 (6), 561.

(11) Xiao, H.; Wu, R. Quantitative investigation of human cell surface N-glycoprotein dynamics. Chemical science 2017, 8 (1), 268.

(12) Sun, S.; Shah, P.; Eshghi, S. T.; Yang, W.; Trikannad, N.; Yang, S.; Chen, L.; Aiyetan, P.; Hoti, N.; Zhang, Z.et al. Comprehensive analysis of protein glycosylation by solid-phase extraction of $\mathrm{N}$ linked glycans and glycosite-containing peptides. Nature biotechnology 2016, 34 (1), 84.

(13) Riley, N. M.; Hebert, A. S.; Westphall, M. S.; Coon, J. J. Capturing site-specific heterogeneity with large-scale N-glycoproteome analysis. Nature communications 2019, 10 (1), 1311.

(14) Liu, M. Q.; Zeng, W. F.; Fang, P.; Cao, W. Q.; Liu, C.; Yan, G. Q.; Zhang, Y.; Peng, C.; Wu, J. Q.; Zhang, X. J.et al. pGlyco 2.0 enables precision $\mathrm{N}$-glycoproteomics with comprehensive quality control and one-step mass spectrometry for intact glycopeptide identification. Nature communications 2017, 8 (1), 438.

(15) Stadlmann, J.; Taubenschmid, J.; Wenzel, D.; Gattinger, A.; Durnberger, G.; Dusberger, F.; Elling, U.; Mach, L.; Mechtler, K.; Penninger, J. M. Comparative glycoproteomics of stem cells identifies new players in ricin toxicity. Nature 2017, 549 (7673), 538.

(16) Mayampurath, A.; Song, E.; Mathur, A.; Yu, C. Y.; Hammoud, Z.; Mechref, Y.; Tang, H. Label-free glycopeptide quantification for biomarker discovery in human sera. Journal of proteome research 2014, 13 (11), 4821. 
(17) Pinho, S. S.; Reis, C. A. Glycosylation in cancer: mechanisms and clinical implications. Nature reviews. Cancer 2015, 15 (9), 540.

(18) Rodriguez, E.; Schetters, S. T. T.; van Kooyk, Y. The tumour glyco-code as a novel immune checkpoint for immunotherapy. Nat Rev Immunol 2018, 18 (3), 204.

(19) Sato, Y.; Nakata, K.; Kato, Y.; Shima, M.; Ishii, N.; Koji, T.; Taketa, K.; Endo, Y.; Nagataki, S. Early recognition of hepatocellular carcinoma based on altered profiles of alpha-fetoprotein. The New England journal of medicine 1993, 328 (25), 1802.

(20) Liu, Y. C.; Yen, H. Y.; Chen, C. Y.; Chen, C. H.; Cheng, P. F.; Juan, Y. H.; Chen, C. H.; Khoo, K. H.; Yu, C. J.; Yang, P. C.et al. Sialylation and fucosylation of epidermal growth factor receptor suppress its dimerization and activation in lung cancer cells. Proceedings of the National Academy of Sciences of the United States of America 2011, 108 (28), 11332.

(21) Potapenko, I. O.; Haakensen, V. D.; Luders, T.; Helland, A.; Bukholm, I.; Sorlie, T.; Kristensen, V. N.; Lingjaerde, O. C.; Borresen-Dale, A. L. Glycan gene expression signatures in normal and malignant breast tissue; possible role in diagnosis and progression. Molecular oncology 2010, 4 (2), 98.

(22) Cao, L.; Diedrich, J. K.; Kulp, D. W.; Pauthner, M.; He, L.; Park, S. R.; Sok, D.; Su, C. Y.; Delahunty, C. M.; Menis, S.et al. Global site-specific N-glycosylation analysis of HIV envelope glycoprotein. Nature communications 2017, 8, 14954.

(23) Unione, L.; Lenza, M. P.; Arda, A.; Urquiza, P.; Lain, A.; Falcon-Perez, J. M.; Jimenez-Barbero, J.; Millet, O. Glycoprofile Analysis of an Intact Glycoprotein As Inferred by NMR Spectroscopy. ACS central science 2019, 5 (9), 1554.

(24) Maley, F.; Trimble, R. B.; Tarentino, A. L.; Plummer, T. H. Characterization of Glycoproteins and Their Associated Oligosaccharides through the Use of Endoglycosidases. Anal Biochem 1989, 180 (2), 195.

(25) Collin, M.; Olsen, A. EndoS, a novel secreted protein from Streptococcus pyogenes with endoglycosidase activity on human IgG. Embo J 2001, 20 (12), 3046.

(26) Trimble, R. B.; Tarentino, A. L. Identification of Distinct Endoglycosidase (Endo) Activities in Flavobacterium-Meningosepticum - Endo-F1, Endo-F2, and Endo-F3 - Endo-F1 and Endo-H Hydrolyze Only High Mannose and Hybrid Glycans. J Biol Chem 1991, 266 (3), 1646.

(27) Clark, P. M.; Dweck, J. F.; Mason, D. E.; Hart, C. R.; Buck, S. B.; Peters, E. C.; Agnew, B. J.; HsiehWilson, L. C. Direct in-gel fluorescence detection and cellular imaging of O-GICNAc-modified proteins. Journal of the American Chemical Society 2008, 130 (35), 11576.

(28) Szychowski, J.; Mahdavi, A.; Hodas, J. J.; Bagert, J. D.; Ngo, J. T.; Landgraf, P.; Dieterich, D. C.; Schuman, E. M.; Tirrell, D. A. Cleavable biotin probes for labeling of biomolecules via azidealkyne cycloaddition. Journal of the American Chemical Society 2010, 132 (51), 18351.

(29) Arnold, J. N.; Wormald, M. R.; Sim, R. B.; Rudd, P. M.; Dwek, R. A. The impact of glycosylation on the biological function and structure of human immunoglobulins. Annual review of immunology 2007, 25, 21.

(30) Tarentino, A. L.; Plummer, T. H., Jr. Enzymatic deglycosylation of asparagine-linked glycans: purification, properties, and specificity of oligosaccharide-cleaving enzymes from Flavobacterium meningosepticum. Methods in enzymology 1994, 230, 44.

(31) Huang, C. C.; Harada, Y.; Hosomi, A.; Masahara-Negishi, Y.; Seino, J.; Fujihira, H.; Funakoshi, Y.; Suzuki, T.; Dohmae, N.; Suzuki, T. Endo-beta-N-acetylglucosaminidase forms N-GlcNAc protein aggregates during ER-associated degradation in Ngly1-defective cells. Proceedings of the 
National Academy of Sciences of the United States of America 2015, 112 (5), 1398.

(32) Khatri, K.; Pu, Y.; Klein, J. A.; Wei, J.; Costello, C. E.; Lin, C.; Zaia, J. Comparison of Collisional and Electron-Based Dissociation Modes for Middle-Down Analysis of Multiply Glycosylated Peptides. Journal of the American Society for Mass Spectrometry 2018, 29 (6), 1075.

(33) Hamouda, H.; Kaup, M.; Ullah, M.; Berger, M.; Sandig, V.; Tauber, R.; Blanchard, V. Rapid analysis of cell surface $\mathrm{N}$-glycosylation from living cells using mass spectrometry. Journal of proteome research 2014, 13 (12), 6144.

(34) Moremen, K. W.; Tiemeyer, M.; Nairn, A. V. Vertebrate protein glycosylation: diversity, synthesis and function. Nature reviews. Molecular cell biology 2012, 13 (7), 448.

(35) Oates, A. J.; Schumaker, L. M.; Jenkins, S. B.; Pearce, A. A.; DaCosta, S. A.; Arun, B.; Ellis, M. J. The mannose 6-phosphate/insulin-like growth factor 2 receptor (M6P/IGF2R), a putative breast tumor suppressor gene. Breast cancer research and treatment 1998, 47 (3), 269.

(36) Ponniah, G.; Nowak, C.; Gonzalez, N.; Miano, D.; Liu, H. Detection and quantitation of low abundance oligosaccharides in recombinant monoclonal antibodies. Analytical chemistry 2015, $87(5), 2718$.

(37) Zheng, K.; Yarmarkovich, M.; Bantog, C.; Bayer, R.; Patapoff, T. W. Influence of glycosylation pattern on the molecular properties of monoclonal antibodies. mAbs 2014, 6 (3), 649.

(38) Rivers, J.; McDonald, L.; Edwards, I. J.; Beynon, R. J. Asparagine deamidation and the role of higher order protein structure. Journal of proteome research 2008, 7 (3), 921.

(39) Hu, H.; Khatri, K.; Klein, J.; Leymarie, N.; Zaia, J. A review of methods for interpretation of glycopeptide tandem mass spectral data. Glycoconjugate journal 2016, 33 (3), 285.

(40) Stavenhagen, K.; Hinneburg, H.; Thaysen-Andersen, M.; Hartmann, L.; Varon Silva, D.; Fuchser, J.; Kaspar, S.; Rapp, E.; Seeberger, P. H.; Kolarich, D. Quantitative mapping of glycoprotein micro-heterogeneity and macro-heterogeneity: an evaluation of mass spectrometry signal strengths using synthetic peptides and glycopeptides. Journal of mass spectrometry : JMS 2013, $48(6)$, i.

(41) Marx, V. Metabolism: sweeter paths in glycoscience. Nature methods 2017, 14 (7), 667.

(42) Rexach, J. E.; Rogers, C. J.; Yu, S. H.; Tao, J.; Sun, Y. E.; Hsieh-Wilson, L. C. Quantification of Oglycosylation stoichiometry and dynamics using resolvable mass tags. Nature chemical biology 2010, 6 (9), 645.

(43) Li, L.; Abou-Samra, E.; Ning, Z.; Zhang, X.; Mayne, J.; Wang, J.; Cheng, K.; Walker, K.; Stintzi, A.; Figeys, D. An in vitro model maintaining taxon-specific functional activities of the gut microbiome. Nature communications 2019, 10 (1), 4146. 